\title{
Probing Secondary Structures of Spin-Labeled RNA by Pulsed EPR Spectroscopy**
}

\author{
Giuseppe Sicoli, Falk Wachowius, Marina Bennati,* and Claudia Höbartner*
}

The ability of RNA to interconvert between multiple conformational states is essential for the diversity of biological functions that have been discovered in the recent past. ${ }^{[1]}$ For example, the correct operation of regulatory RNA elements, such as riboswitches, is based on the precise interplay of alternative RNA conformations. ${ }^{[2]}$ Studying the molecular mechanisms of RNA function entails probing RNA-folding intermediates on the energy landscape. EPR spectroscopy, in particular, has been increasingly applied to obtain structural information on nucleic acids, including local conformational changes in $\mathrm{RNA}^{[3]}$ and the identification of metal-ion binding sites. ${ }^{[4]}$ Pulsed EPR techniques (PELDOR/ DEER) have been used to determine distances between paramagnetic centers in specifically modified RNA. ${ }^{[5]}$ PELDOR should therefore be suitable for the detection of alternative RNA conformations that involve distinct changes in base-pairing patterns.

The accessibility of spin-labeled RNA still poses the major challenge for the widespread applicability of powerful EPR techniques. Nitroxide radicals are the most commonly used type of paramagnetic labels for nucleic acids. Several methods have been reported for attaching nitroxide groups at internal positions at the ribose, the phosphate backbone, or at nucleobases, often by means of multiatom linkers that provide several unwanted degrees of rotational freedom. ${ }^{[6]}$ Rigid nitroxide spin labels conjugated to the nucleobase or to nucleobase analogues have been reported for DNA. ${ }^{[7]}$ Our RNA spin-labeling approach addresses the direct attachment of nitroxide labels onto RNA nucleobases, such that conformational changes can be directly detected by PELDOR (i.e., by the change in distance between two labeled nucleotides). The nucleobase spin labels used in this study are also designed to preserve the Watson-Crick base-pairing capability of labeled nucleotides and not to interfere with

[*] Dr. G. Sicoli, Dr. M. Bennati

Research group Electron Paramagnetic Resonance

Max Planck Institute for Biophysical Chemistry

Am Fassberg 11, 37077 Göttingen (Germany)

E-mail: marina.bennati@mpibpc.mpg.de

F. Wachowius, Dr. C. Höbartner

Research group Nucleic Acid Chemistry

Max Planck Institute for Biophysical Chemistry

Am Fassberg 11, 37077 Göttingen (Germany)

Fax: (+49) 551-201-1680

E-mail: claudia.hoebartner@mpibpc.mpg.de

[***] Financial support from the Max Planck Society is gratefully acknowledged.

$\square$ Supporting information for this article is available on the WWW under http://dx.doi.org/10.1002/anie.201000713. alternative base-pairing patterns in different RNA conformations.

Here, we describe the installation of nitroxide spin labels on exocyclic amino groups of the RNA nucleobases guanine, adenine, and cytosine (Figure 1) with unprecedented effi-
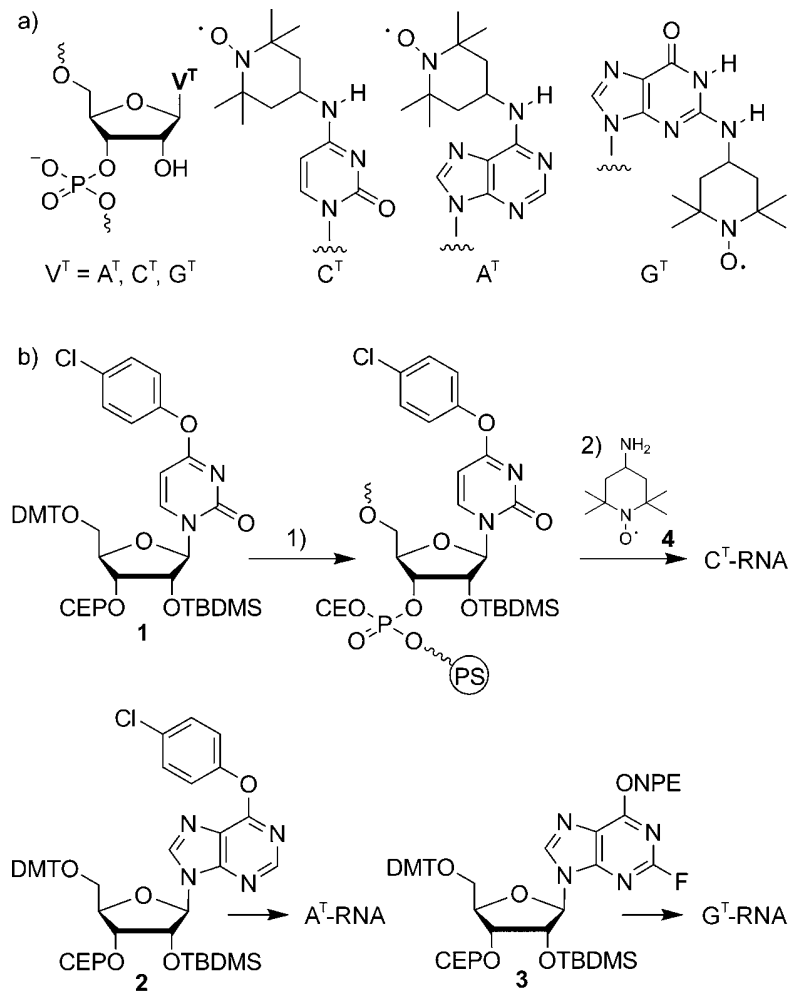

Figure 1. a) Spin-labeled RNA with nitroxide-containing nucleosides $A^{\top}$, $C^{\top}$, and $G^{\top}$. b) Synthesis of spin-labeled RNA using convertible nucleoside phosphoramidites 1-3. 1) Solid-phase synthesis under standard conditions; 2) $2 \mathrm{M} 4$ in methanol, $42^{\circ} \mathrm{C}, 24 \mathrm{~h}$; then $1 \mathrm{M}$ tetrabutylammonium fluoride in $\mathrm{THF}, 25^{\circ} \mathrm{C}, 12 \mathrm{~h}$. DMT $=4,4^{\prime}$-dimethoxytrityl, TBDMS = tert-butyldimethylsilyl, CEP $=(\beta$-cyanoethyl $)$ diisopropylphosphoramidite, NPE =4-nitrophenyl.

ciency, and we report on the evaluation of RNA secondary structures by pulsed double electron resonance spectroscopy.

The convertible nucleosides ${ }^{[8]} O^{4}$-(4-chlorophenyl)uridine, $O^{6}$-(4-chlorophenyl)inosine, and 2-fluoroinosine were incorporated into RNA by solid-phase synthesis using commercially available, modified 2'-O-tert-butyldimethylsilylprotected building blocks 1-3 in combination with standard RNA 2'-O-triisopropylsilyloxymethyl-protected nucleoside phosphoramidites. In a postsynthetic modification step, the 4-chlorophenyl or fluoride leaving groups were displaced by 
incubating the support-bound, fully protected, modified oligonucleotides with 4-amino-2,2,6,6-tetramethylpiperidin-1-oxyl (4) in methanol. Generation of $N^{4}$-TEMPOcytidine $\left(\mathrm{C}^{\mathrm{T}}\right)$ from $\mathrm{O}^{4}$-(4-chlorophenyl)uridine, and $N^{2}$-TEMPO-guanosine $\left(\mathrm{G}^{\mathrm{T}}\right)$ from 2-fluoroinosine, was complete after $24 \mathrm{~h}$ at $42^{\circ} \mathrm{C}$ and no unsubstituted byproducts were observed. The conversion of $O^{6}$-(4chlorophenyl)inosine to $N^{6}$ TEMPO-adenosine $\left(\mathrm{A}^{\mathrm{T}}\right)$ was found to be much slower. 4-Chlorophenyl-containing byproducts were observed even after $96 \mathrm{~h}$ at $42{ }^{\circ} \mathrm{C}$ or $48 \mathrm{~h}$ at $55^{\circ} \mathrm{C} .{ }^{[9]}$ As a general advantage of this spin-labeling strategy, cleavage from the polystyrene support, and deprotection of phosphate backbone and nucleobases occurred at the same time as nucleobase substitution. After removal of the 2'-silyl groups, the TEMPO-substituted RNA oligonucleotides were purified by anion-exchange HPLC or denaturing PAGE. Byproducts from incomplete adenosine substitution could easily be separated. The purified RNAs were characterized by MALDI or ESI-MS. ${ }^{[9]}$

To demonstrate the potential of TEMPO-labeled nucleobases for probing RNA secondary structures, we analyzed the influence of the nitroxide substituent on RNA conformation and the secondary structures of self-complementary RNAs that are able to fold into monomolecular hairpins as well as into bimolecular duplex structures. The self-complementary RNA 5 is known to form a stable double helix over a broad range of concentrations. ${ }^{[10]}$ Various nucleobase modifications that interfere with Watson-Crick base-pairing have been shown to induce a monomolecular hairpin conformation, whereas $\mathrm{N}^{4}$-methylcytidine was well tolerated in the double helix. ${ }^{[10]}$ Similarly, the introduction of a $\mathrm{C}^{\mathrm{T}}$ label in RNA 6 did not change the preferred bimolecular secondary structure. This was demonstrated by examination of the concentration dependence of the melting temperature. ${ }^{[9]}$ Analysis of the UV melting experiments showed a destabilization of $5-6^{\circ} \mathrm{C}$ per TEMPO label at RNA concentrations between 3 and $30 \mu \mathrm{M}$. CD spectra confirmed that the standard A-form of the RNA double helices was not significantly perturbed. ${ }^{[9]}$

The magnitude of the destabilizing effect of $5-6^{\circ} \mathrm{C}$ per spin label was similar for $\mathrm{C}^{\mathrm{T}}-, \mathrm{A}^{\mathrm{T}}$-, and $\mathrm{G}^{\mathrm{T}}$-containing RNAs, as demonstrated by incorporation at different positions in RNA duplexes 20 base pairs (bp) in length (Table 1, RNAs 7 13). The presence of these spin labels in short stem-loop structures showed more pronounced sequence-specific effects. ${ }^{[9]} \mathrm{A}$ stronger destabilization of up to $10^{\circ} \mathrm{C}$ per label was observed for $\mathrm{C}^{\mathrm{T}}$ and $\mathrm{A}^{\mathrm{T}}$ in loop-closing base pairs and in internal base pairs. In contrast, double incorporation of $\mathrm{G}^{\mathrm{T}}$ at two neighboring base pairs led to an increase of $4^{\circ} \mathrm{C}$ for the monomolecular melting temperature.
We employed continuous-wave (CW) EPR spectroscopy to characterize the different spin-labeled RNA species. The CW EPR spectra (Figure $2 \mathrm{a}$ ) indicated fast correlation times and high mobility of the spin labels. Inspection of the line shape suggested that the spectra are composed of at least two contributions with different correlation times, which may be related to different relative mobilities of each spin probe.

The PELDOR pulse sequence was used to measure the distance between two spin labels in double-helical RNA. The RNA oligonucleotides 9-12 each contain two TEMPOlabeled nucleotides at specified positions; the parent RNA 8 is unmodified (see Table 1). Formation of bimolecular
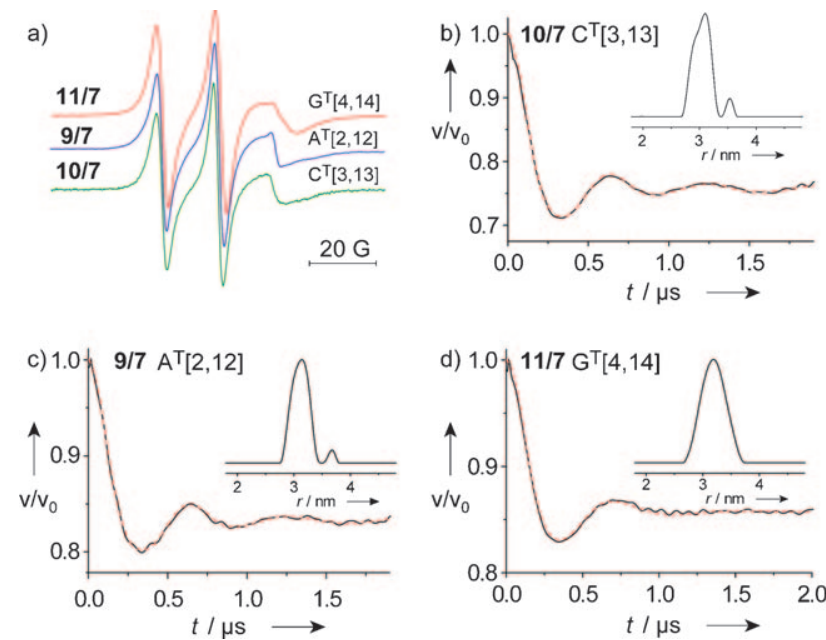

Figure 2. a) $C W$ EPR and b-d) four-pulse PELDOR experiments on doubly TEMPO-labeled RNA duplexes containing $G^{\top}, A^{\top}$, or $C^{\top} .20 \mu \mathrm{M}$ RNA, $10 \mathrm{~mm}$ sodium phosphate buffer, $\mathrm{pH} 7.0,150 \mathrm{~mm} \mathrm{NaCl}$. The modulation depth for the PELDOR traces was registered with a soft pump pulse (100 scans). Dipolar evolution curve (black) and fit (red) obtained by DEERAnalysis $2008 .^{[9]}$ 
duplexes upon hybridization of 9-12 with the complementary RNA 7 leads to a $10 \mathrm{bp}$ separation between two TEMPO groups. The dipolar evolution traces for the RNA duplexes 9/ 7, 10/7, and 11/7 are depicted in Figure $2 b-d$, and the distance distributions obtained by fits with Tikhonov regularization ${ }^{[11]}$ are shown in the insets. The measured distances are in good qualitative agreement with values expected based on idealized A-form duplexes. ${ }^{[9]}$ Despite the high mobility of the spin labels (as deduced from CW EPR data), the experimental distances obtained by pulsed EPR spectroscopy show a narrow distribution, with a half width at half-height of $0.2 \mathrm{~nm}$ for $\mathrm{C}^{\mathrm{T}}$ - and $\mathrm{A}^{\mathrm{T}}$-RNA (Figure $2 \mathrm{~b}, \mathrm{c}$ ), and $0.3 \mathrm{~nm}$ for $\mathrm{G}^{\mathrm{T}}$-RNA (Figure $2 \mathrm{~d}$ ). This most likely arises from the preferred conformation of the spin probes in the deep major groove of A-form RNA duplexes. The slightly broader distance distribution for the $\mathrm{G}^{\mathrm{T}}$-labeled RNA might reflect the higher mobility of the TEMPO group in the shallow minor groove of A-form RNA. The analogous TEMPO-G derivative in a DNA duplex was recently shown to be strongly immobilized in the minor groove of a B-form DNA duplex (full width at half-maximum $0.2 \mathrm{~nm}$ ) but slightly more flexible in the Aform double helix (full width at half-maximum $0.3 \mathrm{~nm}$ ). ${ }^{[12]}$ The modulation depths observed when a strong pump pulse of $12 \mathrm{~ns}$ is used are consistent with those reported for other double-labeled RNA systems. ${ }^{[9,13]}$ The asymmetry in the distance distribution peak of the $\mathrm{C}^{\mathrm{T}}$ - and $\mathrm{A}^{\mathrm{T}}$-labeled RNA duplexes could reflect more than one conformation of the spin label in the major groove of the double helix, which would be consistent with the observation of more than one spectral component in the CW spectra. Alternatively, additional peaks in the distance distribution could result from orientational selectivity ${ }^{[14]}$ of the pump and probe pulses. To investigate this further, PELDOR traces were recorded at different positions of the EPR line. ${ }^{[9]}$ The Fourier transformations of these traces show some dependence on the magnetic field position, indicating orientation selection. However, an orientation-averaging experiment ${ }^{[14 \mathrm{~d}]}$ did not show any change of the distance distribution pattern. ${ }^{[9]}$ Detailed EPR analyses at higher frequency bands (34 or $94 \mathrm{GHz}$ ) are required to extract additional information about relative orientations of nitroxides in TEMPO-labeled RNA. ${ }^{[14]}$

In addition to duplex and hairpin RNA conformations, we analyzed the influence of nucleobase-attached TEMPO spin labels on RNA quadruplex conformations. Guanine quartets in RNA have been studied in the context of telomere transcription, ${ }^{[15]}$ and putative quadruplex-forming sequences have been proposed in untranslated mRNA regions. ${ }^{[16]}$ Here, we studied a model of the human telomeric RNA repeat sequence 14. The solution structure of the bimolecular parallel-stranded G-quadruplex of this 12-mer RNA was recently solved by NMR spectroscopy. ${ }^{[17]}$ Inspection of the lowest-energy structures suggested that TEMPO labels should be well tolerated in the trinucleotide loop region (Figure $3 \mathrm{a}$ ). As expected, the $\mathrm{A}^{\mathrm{T}}$-containing RNA 15 formed a stable quadruplex in the presence of $\mathrm{K}^{+}$ions, analogous to the unmodified RNA 14. This was confirmed by UV melting experiments showing the characteristic hypochromic effect at $295 \mathrm{~nm}$ (Figure $3 \mathrm{~b}$ ). The inflection points of heating or
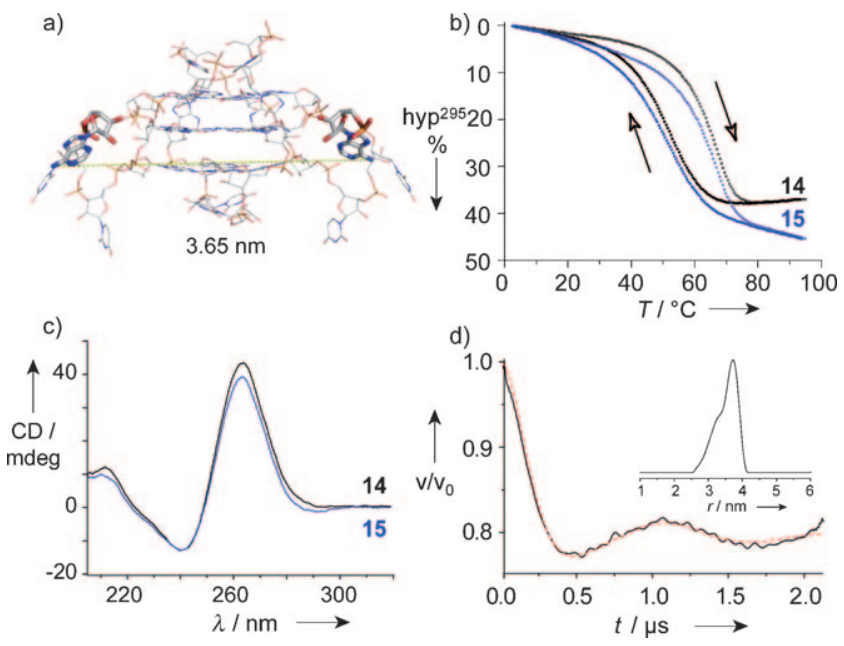

Figure 3. a) Bimolecular RNA quadruplex structure of RNA 14 based on Ref. [17] [PDB: 2KBP]. Distance between attachment sites at the N6 position of adenosines is indicated. b) UV melting curve; hypochromicity at $295 \mathrm{~nm}$ and c) CD spectra of RNA 14 and 15: $7 \mu \mathrm{M}$ RNA, $20 \mathrm{~mm}$ potassium phosphate buffer, $\mathrm{pH} 7.0,70 \mathrm{mM} \mathrm{KCl}$, heating and cooling at $0.2^{\circ} \mathrm{C} \mathrm{min}^{-1}$. d) Dipolar evolution and distance distribution obtained from PELDOR experiment of RNA 15: $20 \mu \mathrm{M}$ RNA, same buffer as for UV and CD $+15 \%$ glycerol.

cooling curves of $\mathbf{1 4}$ and $\mathbf{1 5}$ differed by only $1-2^{\circ} \mathrm{C}$. The circular dichroism spectra for labeled and unlabeled RNA were nearly superimposable (Figure 3c). The distance between the two spin labels in the quadruplex structure was measured by PELDOR. The experimental value of $3.7 \pm$ $0.2 \mathrm{~nm}$ was in good agreement with the distance of $3.65 \mathrm{~nm}$ between the attachment sites found in the NMR structure of the unmodified RNA (Figure 3a,d), although the broader distance distribution may be produced by more than one preferred conformation of the spin labels. Thus, the measured distances strongly support the formation of a parallelstranded quadruplex structure that is consistent with the reported NMR data. ${ }^{[9]}$ In contrast to RNA quadruplexes, model constructs of human telomeric DNA repeats have long been discussed to exist in alternative parallel and antiparallel quadruplex conformations. This was recently confirmed by pulsed EPR measurements of spin-labeled DNA. ${ }^{[18]}$

The promising PELDOR results for various RNA secondary structures and the observed narrow distance distributions obtained for RNAs labeled with $\mathrm{C}^{\mathrm{T}}, \mathrm{A}^{\mathrm{T}}$, and $\mathrm{G}^{\mathrm{T}}$ prompted us to explore the simultaneous detection of alternative RNA structures, such as competing hairpin and duplex conformations of partially self-complementary RNAs. In our model system, the unmodified RNA 8 forms a stable hairpin conformation with a 6 bp stem (underlined nucleotides in the Table), an extrastable GGAA tetraloop and a 3'overhang four nucleotides in length. In the presence of the fully complementary (unmodified) oligonucleotide $\mathbf{7}$, the stem is disrupted and a continuous 20 bp duplex is formed. Sequence 7 by itself also forms a stem-loop structure, and has a $5^{\prime}$-overhang four nucleotides in length. ${ }^{[9]}$ Duplex formation between $\mathbf{7}$ and $\mathbf{8}$ is likely initiated by strand invasion starting from the complementary overhang regions, or by a kissingloop interaction. For both sequences $\mathbf{7}$ and $\mathbf{8}$, the formation of 
a homodimer structure with an internal bulge of four mismatched nucleotides is conceivable, but such homodimers were not observed under our experimental conditions in UV melting experiments and native gel electrophoresis. ${ }^{[9]}$

The secondary structures of the model system $\mathbf{7 / 8}$ were analyzed in the spin-labeled version: The doubly $\mathrm{C}^{\mathrm{T}}$-labeled RNA 12 ("spin-labeled $\mathbf{8}$ ") was used for detailed investigation of hairpin and duplex conformations by PELDOR experiments (Figure 4). In the hairpin conformation of RNA 12, the
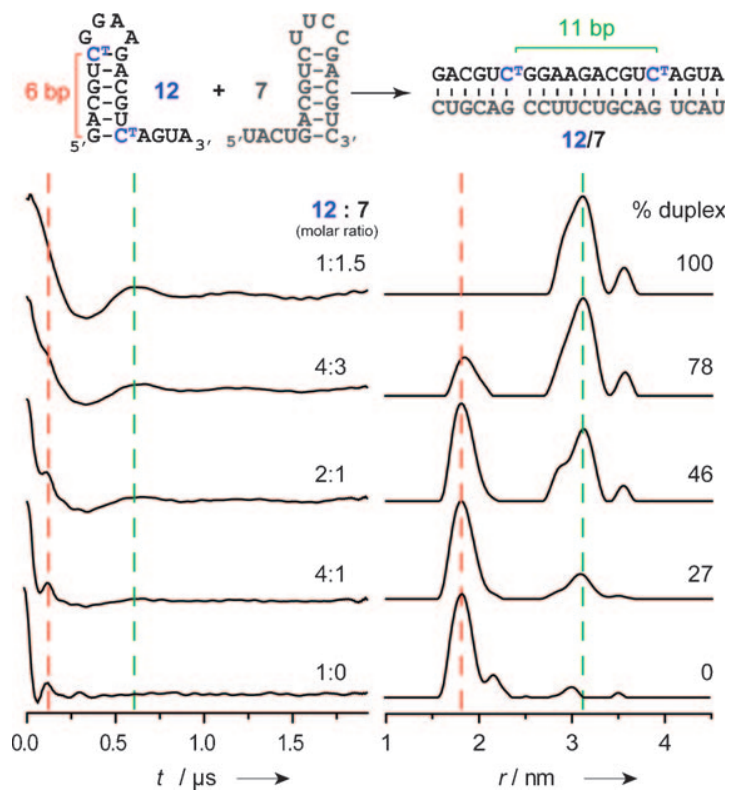

Figure 4. RNAs 12 and 7 individually fold into hairpin conformations but form an extended duplex when mixed. PELDOR titration experiment: stepwise addition of hairpin 7 to hairpin 12 generates 25,50 , 75 , and $100 \%$ duplex in the mixture (duplex content increases from bottom to top). Area integration of the distance distribution allows quantification to $\pm 5 \%$ (experimental values are given in the plot). The small peaks at 2.1, 2.9, and $3.6 \mathrm{~nm}$ are unrelated to hairpin and duplex secondary structures and are assigned to residual ${ }^{14} \mathrm{~N}$ and ${ }^{31} \mathrm{P}$ hyperfine coupling (ESEEM effect).

two TEMPO groups are $6 \mathrm{bp}$ apart. This is reflected in a distance distribution centered at $1.8 \mathrm{~nm}$. In the duplex state, the two TEMPO groups are $11 \mathrm{bp}$ apart, resulting in a distance distribution centered at $3.1 \mathrm{~nm}$. Upon titration of hairpin 12 with increasing amounts of the complementary RNA 7, hairpin and extended duplex conformations coexist in different ratios. This is observed by the corresponding change of the areas under the curve for the two major peaks in the distance distribution, which correlate well with the predicted hairpin/duplex ratios. The hairpin conformation of unlabeled RNA 8 and $C^{\mathrm{T}}$-labeled counterparts $\mathbf{1 2}$ and $\mathbf{1 3}$, as well as the coexistence of hairpin and duplex structures in the presence of complementary RNA 7, was also confirmed by gel shift analysis on native polyacrylamide gels. ${ }^{[9]}$

In summary, we have demonstrated a very efficient way for preparing high-quality spin-labeled RNA for PELDOR experiments. Direct attachment of the TEMPO group to the exocyclic amino groups of nucleobases $\mathrm{A}$ and $\mathrm{C}$ in the major groove, and of $\mathrm{G}$ in the minor groove of Watson-Crick basepaired RNA duplexes has been achieved by the convertible nucleoside approach. ${ }^{[19]}$ This strategy complements other approaches for the preparation of spin-labeled RNA that have been demonstrated for base-specific labeling of uridine, cytidine, and adenosine. ${ }^{[20]}$ To our knowledge, the $N^{2}$ TEMPO-labeled guanosine $\mathrm{G}^{\mathrm{T}}$ is the first guanosine-specific spin label for RNA. ${ }^{[21]}$ All three TEMPO-substituted nucleotides reported in this study proved their potential for application as powerful reporter probes for the determination of alternative secondary structures of RNA by pulsed EPR spectroscopy. We consider the simplicity of the synthetic approach, and the good yield and quality of our spin-labeled RNAs as the major advantages of this novel strategy. The narrow distance distributions obtained from the high-quality PELDOR data of $\mathrm{A}^{\mathrm{T}}$-, $\mathrm{C}^{\mathrm{T}}$-, and $\mathrm{G}^{\mathrm{T}}$-modified RNAs hold great promise for studying conformational rearrangements in larger RNAs and RNA-protein interactions in biologically important ribonucleoprotein complexes.

Received: February 5, 2010

Revised: March 18, 2010

Published online: July 21, 2010

Keywords: EPR spectroscopy - PELDOR - RNA .

secondary structures $\cdot$ spin labeling

[1] a) M. Nowotny, W. Yang, Curr. Opin. Struct. Biol. 2009, 19, 286; b) J. E. Wilusz, H. Sunwoo, D. L. Spector, Genes Dev. 2009, 23, 1494.

[2] A. Serganov, Curr. Opin. Struct. Biol. 2009, 19, 251.

[3] X. J. Zhang, P. Cekan, S. T. Sigurdsson, P. Z. Qin, Methods Enzymol. 2009, 469, 303.

[4] a) T. E. Edwards, S. T. Sigurdsson, Biochemistry 2005, 44, 12870; b) N. Kisseleva, S. Kraut, A. Jäschke, O. Schiemann, HFSP J. 2007, $1,127$.

[5] a) O. Schiemann, A. Weber, T. E. Edwards, T. F. Prisner, S. T. Sigurdsson, J. Am. Chem. Soc. 2003, 125, 3434; b) O. Schiemann, T. F. Prisner, Q. Rev. Biophys. 2007, 40, 1; c) O. Schiemann, Methods Enzymol. 2009, 469, 329.

[6] a) G. Z. Sowa, P. Z. Qin, Prog. Nucleic Acid Res. Mol. Biol. 2008, 82, 147; b) T. E. Edwards, S. T. Sigurdsson, Nat. Protoc. 2007, 2, 1954 ; c) P. Z. Qin, I. S. Haworth, Q. Cai, A. K. Kusnetzow, G. P. G. Grant, E. A. Price, G. Z. Sowa, A. Popova, B. Herreros, H. He, Nat. Protoc. 2007, 2, 2354; d) O. Schiemann, N. Piton, J. Plackmeyer, B. E. Bode, T. F. Prisner, J. W. Engels, Nat. Protoc. 2007, 2, 904.

[7] a) T. R. Miller, S. C. Alley, A. W. Reese, M. S. Solomon, W. V. McCallister, C. Mailer, B. H. Robinson, P. B. Hopkins, J. Am. Chem. Soc. 1995, 117, 9377; b) N. Barhate, P. Cekan, A. P. Massey, S. T. Sigurdsson, Angew. Chem. 2007, 119, 2709; Angew. Chem. Int. Ed. 2007, 46, 2655; c) P. Cekan, A. L. Smith, N. Barhate, B. H. Robinson, S. T. Sigurdsson, Nucleic Acids Res. 2008, 36, 5946.

[8] C. R. Allerson, S. L. Chen, G. L. Verdine, J. Am. Chem. Soc. 1997, 119, 7423.

[9] See the Supporting Information for further details.

[10] R. Micura, W. Pils, C. Höbartner, K. Grubmayr, M. O. Ebert, B. Jaun, Nucleic Acids Res. 2001, 29, 3997.

[11] G. Jeschke, V. Chechik, P. Ionita, A. Godt, H. Zimmermann, J. Banham, C. Timmel, D. Hilger, H. Jung, Appl. Magn. Reson. 2006, 30, 473 . 
[12] G. Sicoli, G. Mathis, O. Delalande, Y. Boulard, D. Gasparutto, S. Gambarelli, Angew. Chem. 2008, 120, 747; Angew. Chem. Int Ed. 2008, 47, 735 .

[13] I. Krstić, O. Frolow, D. Sezer, B. Endeward, J. E. Weigand, B. Suess, J. W. Engels, T. F. Prisner, J. Am. Chem. Soc. 2010, 132, 1454.

[14] a) V. P. Denysenkov, T. F. Prisner, J. Stubbe, M. Bennati, Proc. Natl. Acad. Sci. USA 2006, 103, 13386; b) G. Sicoli, T. Argirevic, J. Stubbe, I. Tkach, M. Bennati, Appl. Magn. Reson. 2010, 37, 539; c) O. Schiemann, P. Cekan, D. Margraf, T. F. Prisner, S. T. Sigurdsson, Angew. Chem. 2009, 121, 3342; Angew. Chem. Int. Ed. 2009, 48, 3292; d) A. Godt, M. Schulte, H. Zimmermann, G. Jeschke, Angew. Chem. 2006, 118, 7722; Angew. Chem. Int. Ed. 2006, 45,7560 .

[15] a) C. M. Azzalin, P. Reichenbach, L. Khoriauli, E. Giulotto, J. Lingner, Science 2007, 318, 798; b) S. Schoeftner, M. A. Blasco, EMBO J. 2009, 28, 2323.
[16] a) S. Kumari, A. Bugaut, J. L. Huppert, S. Balasubramanian, Nat. Chem. Biol. 2007, 3, 218; b) O. Kikin, Z. Zappala, L. D'Antonio, P. S. Bagga, Nucleic Acids Res. 2008, 36, D141.

[17] H. Martadinata, A. T. Phan, J. Am. Chem. Soc. 2009, 131, 2570.

[18] V. Singh, M. Azarkh, T. E. Exner, J. S. Hartig, M. Drescher, Angew. Chem. 2009, 121, 9908; Angew. Chem. Int. Ed. 2009, 48, 9728.

[19] The analogous $A^{T}$ and $C^{T}$ labels for DNA were earlier incorporated by means of phosphoramidite chemistry: a) W. Bannwarth, D. Schmidt, Bioorg. Med. Chem. Lett. 1994, 4, 977; b) C. Giordano, F. Fratini, D. Attanasio, L. Cellai, Synthesis 2001, 565; $C^{\mathrm{T}}$-DNA was recently used for probing single-base mismatches in double-stranded DNA: c) P. Cekan, S. T. Sigurdsson, J. Am. Chem. Soc. 2009, 131, 18054.

[20] N. Piton, Y. Mu, G. Stock, T. F. Prisner, O. Schiemann, J. W. Engels, Nucleic Acids Res. 2007, 35, 3128.

[21] An analogous $G^{T}$ label was earlier incorporated into DNA by a similar displacement strategy: A. Okamoto, T. Inasaki, I. Saito, Bioorg. Med. Chem. Lett. 2004, 14, 3415. 